\title{
Multifocal intrafollicular granulosa cell tumor of the ovary associated with an unusual germline p53 mutation
}

\author{
Francisco F Nogales ${ }^{1}$, Maria L Musto², Ana I Sáez ${ }^{1}$, Mercedes Robledo³ ${ }^{3}$ José Palacios ${ }^{3}$ \\ and José Aneiros ${ }^{1}$ \\ ${ }^{1}$ Departments of Pathology, University Hospital, Granada, Spain; ${ }^{2}$ University of Montevideo, Uruguay \\ and ${ }^{3}$ Centro Nacional de Investigaciones Oncológicas (CNIO), Madrid, Spain
}

\begin{abstract}
A 23-year-old woman presented with a $7 \mathrm{~cm}$ right multicystic mass in the ovary, which corresponded microscopically to an unusual lesion consisting of a multifocal granulosa cell tumor with intrafollicular ('in situ') growth involving two-thirds of mature follicles. Stromal invasion was found in only one area where neoplastic follicles coalesced. Granulosa cells had atypical, bizarre TP53 positive nuclei with hyperchromatism, abundant mitoses and numerous hyaline globules. The contralateral ovary was normal. From the age of 10 years, the patient had a complex medical history of multiple tumors, including telangiectatic osteosarcoma, typical and malignant phyllodes tumor, reticulohistiocytoma of skin, carcinomas of the breast and lipo- and leiomyosarcoma. The female genital tract also harbored myometrial leiomyomas and an early endometrial carcinoma. Retrospective histologic study of all mesenchymal neoplasms in this patient showed, the conspicuous presence of similar bizarre TP53 positive cells with hyaline globules in all the mesenchymal neoplasms. In the genetic study, a germline p53 gene mutation was detected in exon 10, codon 336, generating a stop codon in the oligomerization domain of the protein (E336X). A further p53 mutation was found in exon 7 in the granulosa cell tumor. Mutation occurred de novo since there was no history of tumors in any family members, all of whom had a wild-type p53. Although this patient shows a typical tumor phenotype of Li Fraumeni syndrome, the germline mutation corresponded to a highly unusual mutated domain, which is similar to the one found in childhood malignant adrenocortical tumor; also a rare neoplasm that originates in adrenocortical cells; which are closely related, both functionally and embryologically, to granulosa cells. Modern Pathology (2004) 17, 868-873, advance online publication, 9 April 2004; doi:10.1038/modpathol.3800133
\end{abstract}

Keywords: granulosa cell tumor; p53 gene mutation; Li Fraumeni syndrome; childhood malignant adrenocortical tumor

Germline TP53 mutations are present in Li Fraumeni syndrome (LFS), a rare familial dominant cancer predisposition characterized by the occurrence in children and young adults of a wide spectrum of neoplasms. Analogous mutations may also occur de novo. In these situations, there is a similar clinical association of multiple, characteristic, successive primary tumors of different organs and tissues presenting at a young age. ${ }^{1,6}$ Although mutations may take place throughout the TP53 gene, the majority of cases have occurred in hot spots in exons 5-8. Interestingly, there seems to exist a relationship between the type of mutation (mutational genotype)

Correspondence: Dr FF Nogales MD, Departamento de Anatomía Patológica, Facultad de Medicina, 11, Granada 18012, Spain.

E-mail: fnogales@ugr.es

Received 20 January 2004; accepted 23 February 2004; published online 9 April 2004 and the number and type of cancers and age of presentation (tumor phenotype). ${ }^{3}$

In LFS and related conditions, most reported tumors correspond to sarcomas, early onset breast cancer, brain tumors and childhood adrenocortical carcinoma. Ovarian tumors are rarely reported and they usually correspond to neoplasms of common epithelial type. ${ }^{4}$ To date, no cases of ovarian neoplasms of sex cord-stromal type have been reported associated with TP53 germline mutations. ${ }^{5}$

This paper reports for the first time, the finding of an unusual multifocal follicular neoplastic lesion of granulosa cells in the ovary in a patient with a rare, nonfamilial type of germline mutation of the p53 gene in exon 10, codon 336. This mutation occurred in a domain that is characteristically related to the childhood malignant adrenocortical tumor reported in Brazilian children with $\mathrm{LFS}^{6}{ }^{6}$ a neoplasm with which it shares a common embryogenesis. 


\section{Case history}

This phenotypically normal female was diagnosed at the age of 10 years with a $4 \mathrm{~cm}$ telangiectatic osteosarcoma in the left tibia, involving the surrounding soft tissues and skin and underwent amputation of the left lower limb. After 6 months, she developed pulmonary metastases that were surgically removed and successfully treated with chemotherapy without further recurrence. At the age of 15 years, following the onset of menarche, she developed a small lump in the left breast that was diagnosed as a fibroadenoma. After 3 years, she developed large, bilateral $(8$ and $14 \mathrm{~cm}$ in diameter) mammary tumors that were diagnosed as a malignant phyllodes tumor in the right breast and a histologically benign tumor in the left one. Extensive tumorectomy of both breasts was performed. A recurrence took place in the right breast 3 years later, which was subsequently treated by local excision. At the age of 23 years, the left breast showed a small area of less than $1 \mathrm{~cm}$ corresponding to a high-grade intraductal carcinoma. After 1 year, a further $1 \mathrm{~cm}$ invasive ductal carcinoma was detected, this time in the contralateral breast. A right mastectomy with axillary dissection was performed, revealing three involved lymph nodes. The left breast was also removed some months later due to the presence of a recurrent malignant phyllodes tumor.

At the age of 23 years, a fast growing abdominal mass was detected ultrasonographically, which on laparotomy corresponded to a $7 \mathrm{~cm}$ multicystic mass in the right ovary and was treated by salpingooophorectomy. The contralateral ovary was normal in appearance. After 2 years, the presence of suspicious uterine fundal masses prompted further laparotomy, which revealed intramural leiomyomas.
After informed consent from patient, total hysterectomy with removal of the remaining adnexum was performed. The endometrium proved to harbor an incipient endometrioid adenocarcinoma.

Subsequently, further neoplasms have been detected in this patient: a reticulohistiocytoma of the skin in the nose region diagnosed at 21 years of age and a grade I well-differentiated retroperitoneal liposarcoma at the age of 24 years. The latest event was the presence of an osteolytic lesion in the iliac crest, which was diagnosed in a core biopsy as a leiomyosarcoma. The chronology and clinical features of all successive neoplasms to the present date are summarized in Table 1.

An exhaustive investigation of a family history of cancer is negative in parents, siblings, aunts and uncles.

\section{Pathology}

\section{Female Genital Tract Neoplasms}

The $6 \times 5 \times 5 \mathrm{~cm}$ right ovary had numerous dilated cortical cysts filled with serohemorrhagic contents. Microscopically, only some of the follicles, including primary ones, had a normal histological appearance but more than two-thirds of the follicles at different stages of maturation (from secondary to Graafian), showed a multifocal granulosa cell change (Figure 1) with intraluminal growth consisting in bizarre cells characterized by marked anaplasia, hyperchromatism, abundant mitoses and numerous irregular cytoplasmic and extracellular hyaline globules (Figure 1). Both the theca interna and externa cells of the neoplastic follicles were not involved. The phenotypically altered granulosa cells showed uniform immunohistochemical TP53

Table 1 Chronology, histologic types and clinical features of successive tumours

\begin{tabular}{|c|c|c|c|c|c|}
\hline $\begin{array}{l}\text { Age } \\
\text { (years) }\end{array}$ & Site & Histologic type & Recurrences & Metastases & Treatment \\
\hline 10 & Lt tibia & Telangiectatic osteosarcoma & 6 months & Pulmonary & Amputation and chemotherapy \\
\hline 15 & Lt breast & Fibroadenoma & - & - & Local excision \\
\hline 18 & $\begin{array}{l}\text { Bilateral } \\
\text { breasts }\end{array}$ & $\begin{array}{l}\text { Rt malignant phyllodes tumor } \\
\text { Lt Phyllodes tumor }\end{array}$ & 3 \& 5 years & - & $\begin{array}{l}\text { Extensive local excision } \\
\text { followed by bilateral mastectomy } \\
\text { at } 25 \text { years }\end{array}$ \\
\hline 21 & Skin (nose) & Reticulohistiocytoma & & & Local excision \\
\hline 23 & Lt breast & Intraductal carcinoma & - & - & Local excision \\
\hline 23 & Rt ovary & $\begin{array}{l}\text { Multifocal Intrafollicular } \\
\text { Granulosa cell tumor }\end{array}$ & - & - & $R S O$ \\
\hline 24 & Rt breast & Invasive ductal carcinoma & & 3 axillary nodes & $\begin{array}{l}\text { Rt mastectomy and axillary } \\
\text { dissection }\end{array}$ \\
\hline 24 & Retroperitoneum & Well differentiated liposarcoma & - & - & Local excision \\
\hline 25 & Myometrium & Leiomyomas & - & - & TAH-LSO \\
\hline 25 & Endometrium & $\begin{array}{l}\text { Intramucosal endometrioid } \\
\text { adenocarcinoma with } \\
\text { atypical hyperplasia }\end{array}$ & - & - & TAH-LSO \\
\hline 25 & Iliac bone & Leiomyosarcoma & - & - & Local excision \\
\hline
\end{tabular}

Rt-right, Lt-left 
a

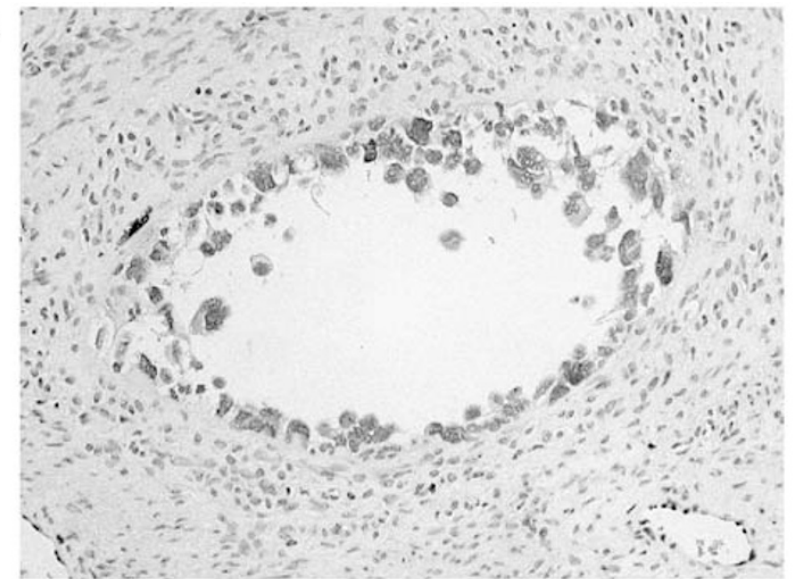

c

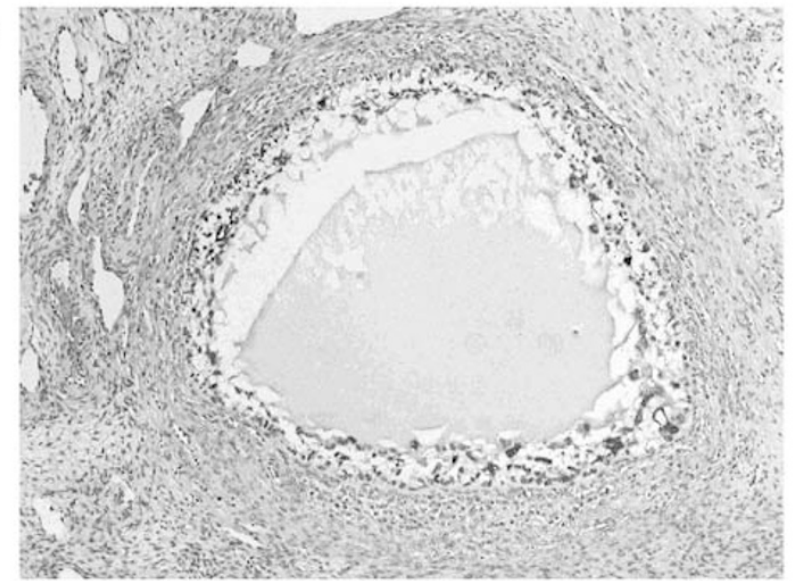

b

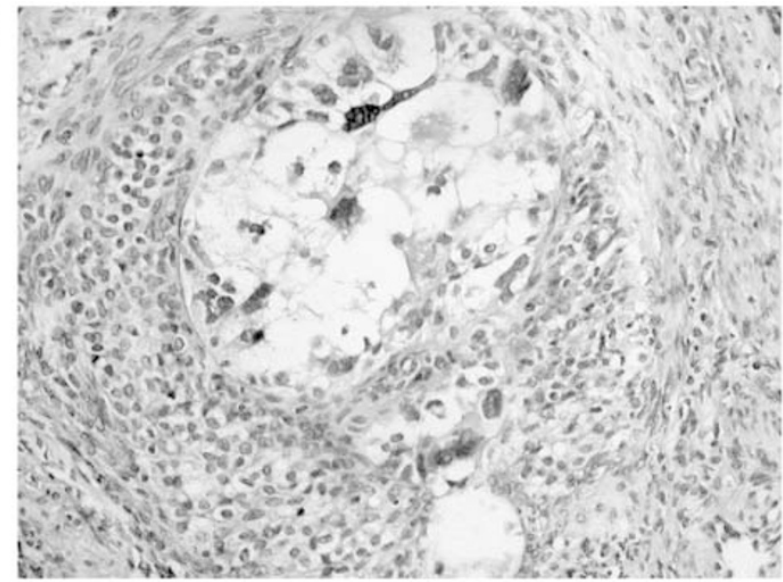

d

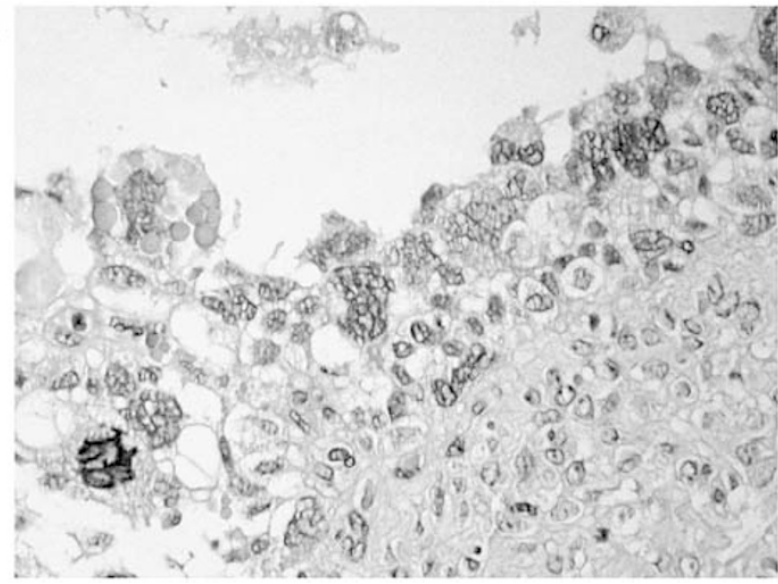

Figure 1 Low power of tertiary follicles with intrafollicular malignant granulosa cell change with bizarre, anaplastic cells (a, b and c). Thecal cells are not involved. Higher magnification (d) showing hyaline intracytoplasmic globules and an atypical mitosis. All H\&E.

positivity, dot-like positivity for CAM 5.2 cytokeratins and diffuse positivity for vimentin, $\alpha$-inhibin and calretinin (All antibodies used in this study from Master Diagnostica, Granada, Spain). Intrafollicular mucin secretion was occasionally found. Proliferative index (measured as Ki67 expression) was moderately high. In some follicles, the granulosa cell clumps were neovascularized and exhibited vascular proliferation. Some of the newly formed vessels were dilated, had fibrous walls and were situated at the core of coarse papillary intrafollicular projections lined by atypical granulosa cells. (Figure 2). Although the change seemed to be predominantly intrafollicular without rupture of the basement membrane, in one area there was a confluence of fused atypical follicular masses that were poorly delineated from the surrounding ovarian parenchyma.

The contralateral ovary extirpated 1 year later was normal. In the hysterectomy specimen, there were two histologically unremarkable fundal leiomyomas. In the endometrium, however, there was a focal lesion less than $4 \mathrm{~mm}$ in size, which corresponded to a complex atypical hyperplasia merging with an early endometrioid adenocarcinoma. The adjoining endometrium was secretory.

\section{Neoplasms of Other Locations}

All tumors, except the ovarian lesion, had the usual features, both macro and microscopically of telangiectatic osteosarcoma, typical and malignant phyllodes tumor, reticulohistiocytoma, intraductal and infiltrating ductal carcinoma of the breast, welldifferentiated liposarcoma and leiomyosarcoma. It is worth noting that a distinctive feature shared by all the mesenchymally derived neoplasms in this patient (phyllodes, osteosarcoma, liposarcoma, leomyosarcoma and granulosa cell tumors) was the presence of abundant, large, scattered, bizarre cells exhibiting marked immunohistochemical TP53 positivity and numerous $\alpha$-1antitrypsin (A1AT) hyaline globules in their cytoplasm (Figure 3). All tumors analyzed, with the exception of reticulohistiocytoma, leiomyomas and intramucosal endometrioid adenocarcinoma, were strongly positive for TP53. 


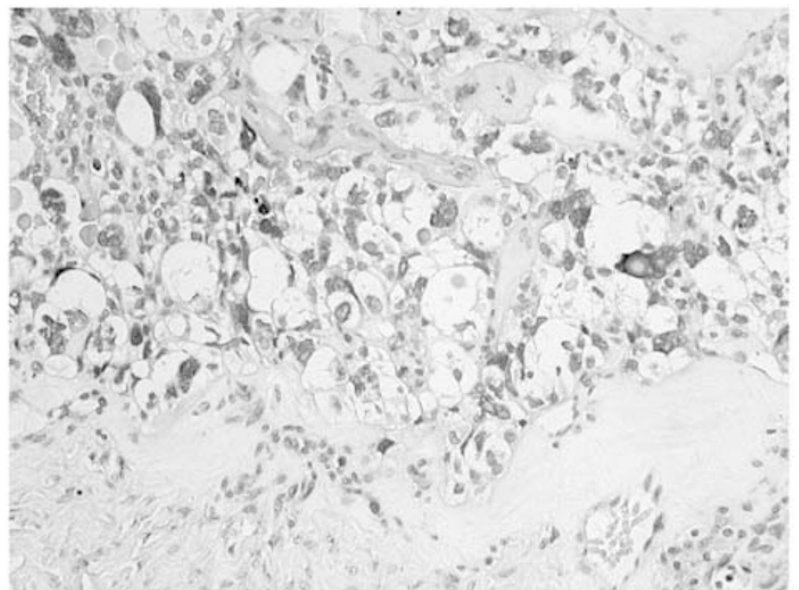

b

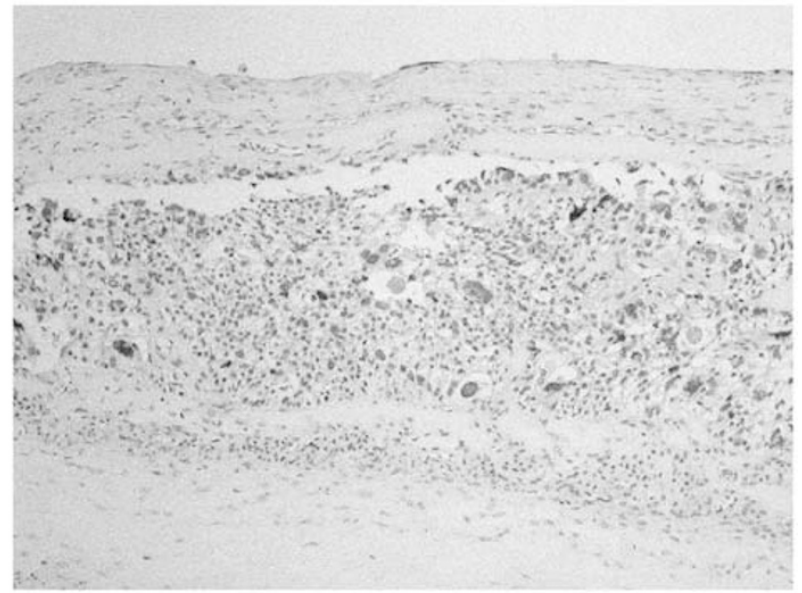

c

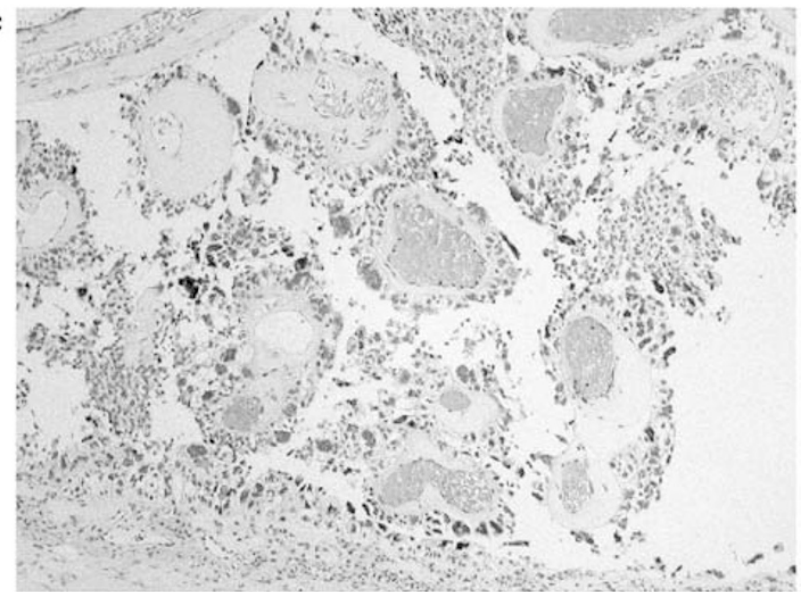

Figure 2 Anaplastic granulosa cells with hyaline globules cells in a follicular wall. Solid intrafollicular tumor with bizarre cells (a, b). Intrafollicular papillary growth with bizarre cell lining and hyalinized vessels (c). All H\&E.

\section{Genetic study}

Genetic studies were performed on blood of patient and relatives (both parents, two siblings, one uncle) and on archival formalin-fixed, paraffin-embedded tissue of the granulosa cell tumor. DNA was
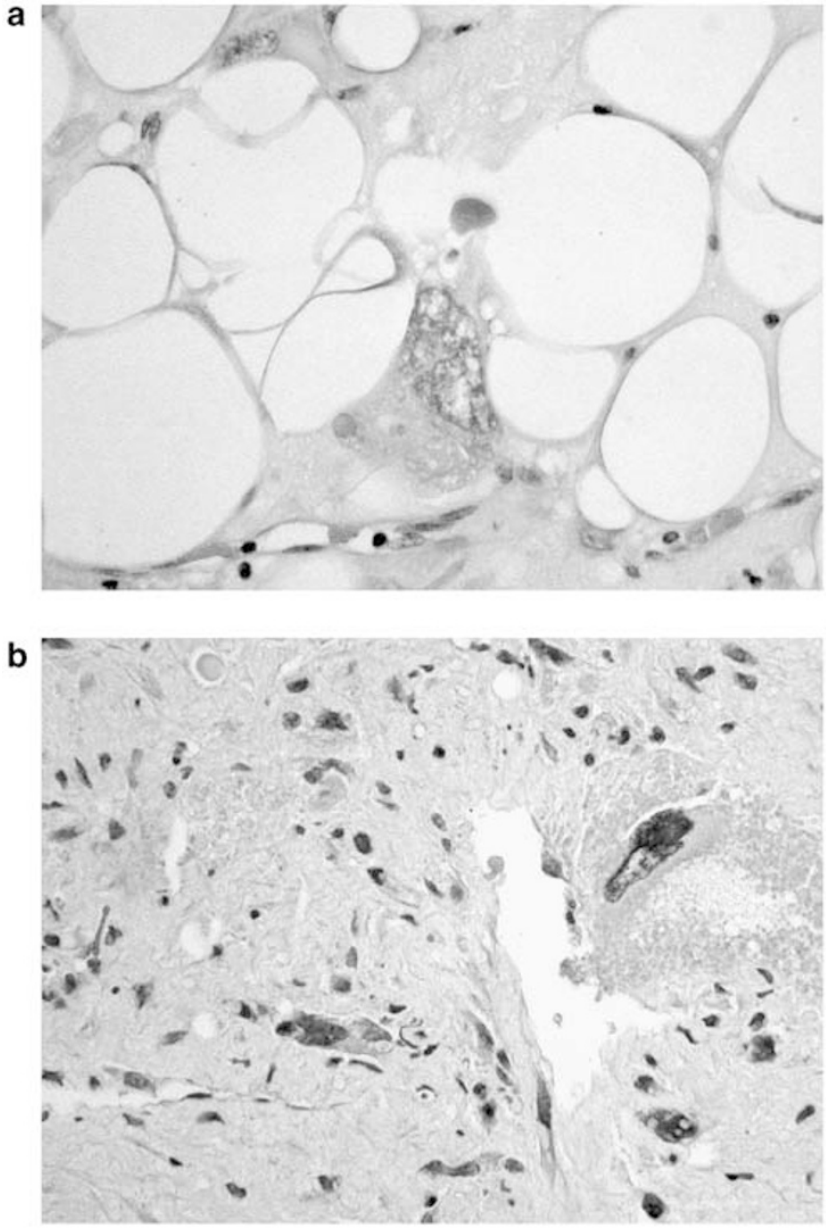

Figure 3 Bizarre cells with hyaline globules in liposarcoma (a) and leiomyosarcoma (b) from the same patient. Both H\&E.

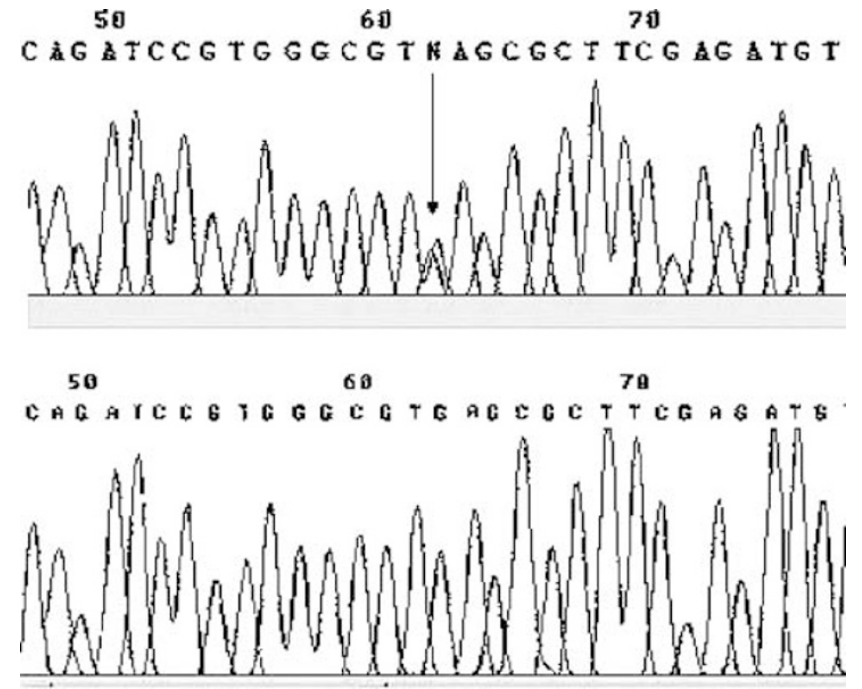

Figure 4 Sequence of germline p53 mutation in exon 10 consisting of a nucleotide change (GAG to TAG) in codon 336 (mutation E336X) (top). The same sequence from the wild-type exon from the father is shown as reference (bottom). 
extracted according to standard protocols using proteinase $\mathrm{K}$ digestion and phenol/chloroform extraction. Mutations in exons 2-11 of p53 were analyzed by direct sequencing with an automated DNA sequencer ABI PRISM 3700 DNA Analyzer (Applied Biosystems) according to the manufacturer's procedures. The primers and conditions for this sequencing have been previously described. ${ }^{7}$

In the patient's blood, a p53 gene mutation was detected consisting of a nucleotide change (GAG to TAG) in codon 336 of exon 10 (Figure 4) generating a stop codon in the oligomerization domain of the protein [E336X]. A further mutation was found in the granulosa cell tumor, consisting in a $\mathrm{T}$ insertion (ATG to TAT) in codon 237 of exon 7. All relatives studied had a wild-type p53 gene. The karyotypes of the patient and her relatives were normal.

\section{Discussion}

This hitherto unreported form of granulosa cell neoplasm presented as a multifocal intrafollicular 'in situ' tumor, synchronously involving many follicles and only focally infiltrating the ovarian stroma. The tumor cells had the phenotype of immature granulosa cells with expression of $\alpha$ inhibin, calretinin and punctuate cytokeratin. They shared with the proliferating cells of the juvenile granulosa cell tumor the presence of extracellular mucin. The conspicuous immunohistochemical positivity for mutant TP53 in the proliferating cells is found in 57-95\% of granulosa cell tumors. ${ }^{8,9}$ Similar positivity was also present in all the sarcomas of this patient in but was absent in reticulohistiocytoma, leiomyomas and intramucosal endometrioid adenocarcinoma.

This patient shows a typical clinical presentation of a full-blown LFS, with four types of characteristic sarcomas (osteo-, lipo-, leiomyosarcoma and malignant phyllodes tumor) and breast cancer all presenting at a young age. This phenotype is associated with mutations within the central core domain of the p53 gene. ${ }^{3}$ However, since this patient has no antecedents of familial tumors or germline mutations in the family, she does not conform with the classical definition of this syndrome ${ }^{1,2}$ and has to be considered as a de novo mutation. Germline p53 mutations have also been reported in families with features suggestive of LFS, but not strictly conforming to the canonical definition. ${ }^{10}$ These families were referred to as either 'LFS-like' or 'incomplete-LFS'. The relative frequence of mutations is $70 \%$ of LFS, $20 \%$ of LFS-like and $6 \%$ of incomplete LFS. Nonfamilial germline mutations such as found in this patient who had no family history of cancer, are unusual. ${ }^{5}$

In LFS, most p53 mutations are located in the highly conserved regions of exons 5-8 in the DNAbinding domain, particularly in exons 7 and $8 .^{3,11,12}$ Other exons may be involved, but less frequently. For this reason, the combination of DNA sequence analysis of the entire coding region of exons 2 through 11 together with complementary immunohistochemical staining is mandatory in order to detect all known alterations in the p53 gene. ${ }^{3}$ The exon 10 mutation present in this patient is highly infrequent. Indeed, in a p53 mutation database, ${ }^{5}$ this mutated exon was present in only $3.5 \%$ of 1181 LFS patients.

Childhood malignant adrenocortical tumor is characteristically associated with $\mathrm{LFS}^{6}$ and usually involves mutations at exon 10 at 'hot spot' 337 only. This dimerization domain (amino acids 336-353) is essential for p53 function and mutations are associated with loss of transactivation activity. ${ }^{3}$ It has been proposed that this mutation occurs in a tissuespecific manner and involves a close relationship between genotype and tumor phenotype. ${ }^{6,13,14}$ If mutations of exon 10 conform tissue specificity, it can be speculated that similar mutational changes may target tissues of closely related embryogenesis such as adrenocortical and granulosa cells, which originate from immediate celomic thickenings at the 6 th week of development. The mutation in the adjoining codon 336 in the present patient supports this notion. Patients with childhood malignant adrenocortical tumor associated with a mutation of exon 10 tend to have an infrequent family history of cancer, although gene carriers were identified in their families. ${ }^{14}$ However, our case presents interesting contrasts: the mutation occurred de novo and its tumor phenotype, although it involved the ovary, was more characteristic of the frequent type of LFS with a mutation of the central core domain of the gene. ${ }^{3}$

p53 germline mutations in female genital tract tumors are relatively infrequent (only $4 \%$ of tumors). ${ }^{5}$ It has been shown that LFS has no increased frequency of female genital tumors; ${ }^{3}$ therefore, the granulosa cell tumor of our patient may be a coincidental finding. However, we believe it to be mutation-related, since it has an unusual presentation characterized by a unique multifocal, intrafollicular distribution and its neoplastic cells share common morphological features with the other sarcomas of this patient. The large bizarre cells and the excess of hyaline globules were such a constant finding as to act as a histologic tumor marker, very likely related to the germline p53 mutation. Hyaline globules are related to apoptosis and cell death, ${ }^{15}$ a phenomenon in which p53 plays an important function. In childhood malignant adrenocortical tumor where a similar mutation occurs in the same dimerization domain of TP53, it is believed that an altered apoptotic function may be responsible for tumorigenesis. ${ }^{6}$ The bizarre nuclei in the presented case are not a common feature of granulosa cell tumours, although this phenomenon has been reported in ovarian sex-cord tumors, ${ }^{16}$ and in some instances of juvenile granulosa cell tumor. ${ }^{17}$

Not all germline p53 mutations in LFS are accompanied by mutation or loss of the wild allele in the tumors, since they may have a gain-offunction or are dominant. ${ }^{3}$ In our case, we detected 
an additional mutation in the ovarian neoplasm, which led to the inactivation of both alleles. Probably, this inactivation in the ovarian tumor facilitated the neoplastic transformation.

Although no similar cases of granulosa cell tumors associated to germline p53 mutations have been reported, there exists a well-known association of juvenile granulosa cell tumors with syndromes involving mesenchymal dysplastic tumors of bone and soft parts, namely Mafucci and Ollier's. ${ }^{18}$ Moreover, a large retrospective analysis of 125 patients with juvenile granulosa cell tumors, ${ }^{17}$ revealed three concomitant cases of osteosarcoma of the leg, maxillary liposarcoma and hemangiopericytoma of bone in young patients. The type of these tumors and their age of presentation are suggestive of LFS. All this indirect evidence suggests that this phenomenon may have been overlooked in the past. Furthermore, occasional alterations of chromosome 17, which is the site of the p53 gene, have been reported. ${ }^{9}$

The biology of the presented ovarian tumor is difficult to ascertain. Although in most follicles the neoplastic change seemed to be restricted by the basal lamina, conforming multiple in situ tumors, there was an area where confluence and fusion of follicles determined stromal invasion. The change respected some follicles in the same ovary and did not involve any in the contralateral one. All mesenchymal tumors in this patient, despite their anaplastic cellular changes, seemed to behave in a not too an aggressive manner. Finally, it is worth commenting on the other neoplastic changes in the female genital tract, such as leiomyomas and endometrioid adenocarcinoma of endometrium. Possibly their relationship could be hormonal rather than mutational, as endometrioid carcinoma does not seem to show p53 mutation as an early carcinogenic event. ${ }^{19}$ Furthermore, the association of granulosa cell tumors with both leiomyomas and endometrioid adenocarcinoma is relatively common due to the increased estrogenic secretion of granulosa cell tumors.

\section{Acknowledgements}

We thank Drs P Torne, PA Clavero, A Isaac (University Hospital, Granada), Rocío Letón and M Urioste (CNIO, Madrid) for their clinical assistance and helpful comments.

\section{References}

1 Birch JM, Alston RD, McNally RJ, et al. Relative frequency and morphology of cancers in carriers of germline TP53 mutations. Oncogene 2001;20: 4621-4628.

2 Li FP, Fraumeni Jr JF. Soft-tissue sarcomas, breast cancer, and other neoplasms. A familial syndrome? Ann Intern Med 1969;71:747-752.

3 Varley JM. Germline TP53 mutations and Li-Fraumeni syndrome. Hum Mutat 2003;21:313-320.

4 Schuijer M, Berns EM. TP53 and ovarian cancer. Hum Mutat 2003;21:285-291.

5 The IARC TP53 mutation database. http://www.iarc.fr/ P53/. Last update: October 282003.

6 Ribeiro RC, Sandrini F, Figueiredo B, et al. An inherited p53 mutation that contributes in a tissuespecific manner to pediatric adrenal cortical carcinoma. Proc Natl Acad Sci USA 2001;98:9330-9335.

7 Urioste M, Martinez-Ramirez A, Cigudosa JC, et al. Complex abnormalities including telomeric associations and MEN1 mutation in a pediatric ependymoma. Cancer Genet Cytogenet 2002;138:107-110.

8 Gebhart JB, Roche PC, Keeney GL, et al. Assessment of inhibin and p53 in granulosa cell tumors of the ovary. Gynecol Oncol 2000;77:232-236.

9 Mayr D, Kaltz-Wittmer C, Arbogast S, et al. Characteristic pattern of genetic aberrations in ovarian granulosa cell tumors. Mod Pathol 2002;15:951-957.

10 Chompret A. The Li-Fraumeni syndrome. Biochimie 2002;84:75-82.

11 Kleihues P, Schauble B, Zur Hausen A, et al. Tumors associated with p53 germline mutations: a synopsis of 91 families. Am J Pathol 1997;150:1-13.

12 Kupryjanczyk J, Thor AD, Beauchamp R, et al. p53 gene mutations and protein accumulation in human ovarian cancer. Proc Natl Acad Sci USA 1993;90: 4961-4965.

13 McDougal WS. An inherited p53 mutation that contributes in a tissue-specific manner to pediatric adrenal cortical carcinoma. J Urol 2002;168:1289.

14 Varley JM, McGown G, Thorncroft M, et al. Are there low-penetrance TP53 Alleles? Evidence from childhood adrenocortical tumors. Am J Hum Genet 1999;55:995-1006.

15 Papadimitriou JC, Drachenberg CB, Brenner DS, et al. 'Thanatosomes': a unifying morphogenetic concept for tumor hyaline globules related to apoptosis. Hum Pathol 2000;31:1455-1465.

16 Young RH, Scully RE. Ovarian sex cord-stromal tumors with bizarre nuclei: a clinicopathologic analysis of 17 cases. Int J Gynecol Pathol 1983;1:325-335.

17 Young RH, Dickersin GR, Scully RE. Juvenile granulosa cell tumor of the ovary. A clinicopathological analysis of 125 cases. Am J Surg Pathol 1984;8: 575-596.

18 Velasco A, Alonso A, Blanco A, et al. Ollier's disease associated with ovarian juvenile granulosa cell tumor. Report of a case and literature review. Cancer 1988;62:222-225.

19 Silverberg SG, Kurman RJ, Nogales FF, et al. Epithelial tumours and related lesions of the uterine corpus. In: Tavassoli FA and Stratton MR (eds). WHO Classification of Tumours. III. Pathology and Genetics of Tumours of the Breast and Female Genital Organs. IARC: Lyon, 2003, pp 230-232. 